\title{
A bio-artificial poly([D,L]-lactide-co-glycolide) drug-eluting nanofibrous periosteum for segmental long bone open fractures with significant periosteal stripping injuries
}

\author{
This article was published in the following Dove Press journal: \\ International Journal of Nanomedicine \\ 8 March 2016 \\ Number of times this article has been viewed
}

\author{
Ying-Chao Chou' ${ }^{1,2}$ \\ Yi-Shiun Cheng' \\ Yung-Heng $\mathrm{Hsu}^{1,2}$ \\ Yi-Hsun $\mathrm{Yu}^{1,2}$ \\ Shih-Jung Liu' ${ }^{1,2}$
}

'Biomaterials Lab, Department of Mechanical Engineering, Chang Gung University, ${ }^{2}$ Department of Orthopedic Surgery, Chang Gung Memorial Hospital, Taoyuan, Taiwan
Correspondence: Shih-Jung Liu Biomaterials Lab, Department of Mechanical Engineering, Chang Gung University, 259, Wen-Hwa Ist Road, Kwei-Shan, Taoyuan 333, Taiwan

Tel +88632118166

Fax +88632118558

Email shihjung@mail.cgu.edu.tw
Abstract: Biodegradable poly([D,L]-lactide-co-glycolide) (PLGA) nanofibrous membrane embedded with two drug-to-polymer weight ratios, namely $1: 3$ and $1: 6$, which comprised PLGA $180 \mathrm{mg}$, lidocaine $20 \mathrm{mg}$, vancomycin $20 \mathrm{mg}$, and ceftazidime $20 \mathrm{mg}$, and PLGA $360 \mathrm{mg}$, lidocaine $20 \mathrm{mg}$, vancomycin $20 \mathrm{mg}$, and ceftazidime $20 \mathrm{mg}$, respectively, was produced as an artificial periosteum in the treatment of segmental femoral fractures. The nanofibrous membrane's drug release behavior was assessed in vitro using high-performance liquid chromatography and the disk-diffusion method. A femoral segmental fracture model with intramedullary Kirschner-wire fixation was established for the in vivo rabbit activity study. Twenty-four rabbits were divided into two groups. Twelve rabbits in group A underwent femoral fracture fixation only, and 12 rabbits in group B underwent femoral fracture fixation and were administered the drug-loaded nanofibers. Radiographs obtained at 2, 6, and 12 weeks postoperatively were used to assess the bone unions. The total activity counts in animal behavior cages were also examined to evaluate the clinical performance of the rabbits. After the animals were euthanized, both femoral shafts were harvested and assessed for their torque strengths and toughness. The daily in vitro release curve for lidocaine showed that the nanofibers eluted effective levels of lidocaine for longer than 3 weeks. The bioactivity studies of vancomycin and ceftazidime showed that both antibiotics had effective and sustained bactericidal capacities for over 30 days. The findings from the in vivo animal activity study suggested that the rabbits with the artificial drug-eluting periosteum exhibited statistically increased levels of activity and better clinical performance outcomes compared with the rabbits without the artificial periosteum. In conclusion, this artificial drug-eluting periosteum may eventually be used for the treatment of open fractures.

Keywords: artificial periosteum, biodegradable poly([D,L]-lactide-co-glycolide) drug-eluting nanofibers, polycaprolactone stents, segmental long bone open fractures

\section{Introduction}

The periosteum is a membrane that covers the entire outer surfaces of all bones, except that of the joints of the long bones. The periosteum consists of dense irregular connective tissue, and it can be divided into an outer fibrous layer and an inner cambium layer. The fibrous layer contains fibroblasts, while the cambium layer contains progenitor cells that develop into osteoblasts. ${ }^{1}$ As a consequence of its high level of vascularization and the presence of osteogenic progenitor cells, osteoinductive growth factors, and an osteoconductive structure, the periosteum 
comprises a critical component of the bone-healing process. ${ }^{2}$ Often, the damaged periosteum cannot be repaired directly during treatment because of the significant stripping injuries and tissue losses that occur in association with open fractures. ${ }^{3}$

The management of severe open fractures poses several challenges for surgeons because open fractures are associated with numerous problems, including infections, tissue defects, and nonunions. ${ }^{4}$ The literature usually recommends suitable initial management of open fractures, which involves copious irrigation and thorough debridement. ${ }^{5}$ The open wound should be covered with an appropriate flap during the early stage to reduce the risk of infection and further tissue loss. ${ }^{6}$ Meanwhile, a suitable bony fixation also plays an important role in the final outcomes from the treatment of open fractures. ${ }^{7}$ The need for the further development of new surgical techniques cannot be overemphasized in relation to the treatment of this hazardous injury. Innovative flap designs and dressing materials have helped surgeons provide early soft tissue coverage. ${ }^{8}$ Modern fixation devices also enable surgeons to offer early functional limb restoration. ${ }^{9}$ Upon trauma, the periosteum might be damaged along with the bone fracture. Furthermore, infections typically occur in fractures because bacteria enter the body during the traumatic event. Few publications address the concerns associated with the management of the periosteum during the treatment of open fractures. ${ }^{10}$

An ideal biomimetic periosteum would provide delivery of osteoinductive growth factors, restore angiogenic potential, and inhibit microbial infection on the bone surface, to allow better bone union. ${ }^{11,12}$ For those open fractures with comminuted fracture fragments, we also expect that the biomimetic periosteum can provide some mechanical strength for seizing the isolated bone fragments to decrease the fragmental irritated fracture pain and decrease nonunion due to excessive micro-motions between fracture fragments.

In this study, we hypothesize that the periosteum can be restored using biomaterial engineering techniques. The aim of this work was to develop an artificial periosteum that incorporates biodegradable drug-embedded nanofibers to provide an adequate drug release capacity, and biodegradable stents to mimic the mechanical properties of the periosteum during the management of open fractures. The in vitro and in vivo effectiveness and safety of the artificial periosteum were examined.

\section{Materials and methods Fabrication of the biodegradable polycaprolactone stent elements}

The biodegradable polymer used in this study was poly ( $\varepsilon$-caprolactone) (PCL) that has a molecular weight of 80,000 Da (Sigma-Aldrich, St Louis, MO, USA). The polymer's density is $1.145 \mathrm{~g} / \mathrm{mL}$ at $25^{\circ} \mathrm{C}$, and it melts at $\sim 60^{\circ} \mathrm{C}$. To fabricate a biodegradable stent, the stent elements were initially produced, each of which consisted of an ellipse connected to two rings at each side. The stent elements were produced using a laboratory-made microinjection molding machine and a mold. ${ }^{13}$ Figure $1 \mathrm{~A}$ displays the dimension of the stent element, while Figure 1B shows the mold used to produce the element. The machine was used to melt, transport, and pressurize the polymer into the molds. The melt temperature was set at $100^{\circ} \mathrm{C}$, and the temperature of the molds was maintained at room temperature. To produce a stent element, the granular form of PCL was fed into the machine, and it was melted. The melted polymer was then squeezed into the core of the mold cavity. Once the stent element had cooled down, the mold was opened, and the stent element was obtained. Each molded stent element was then
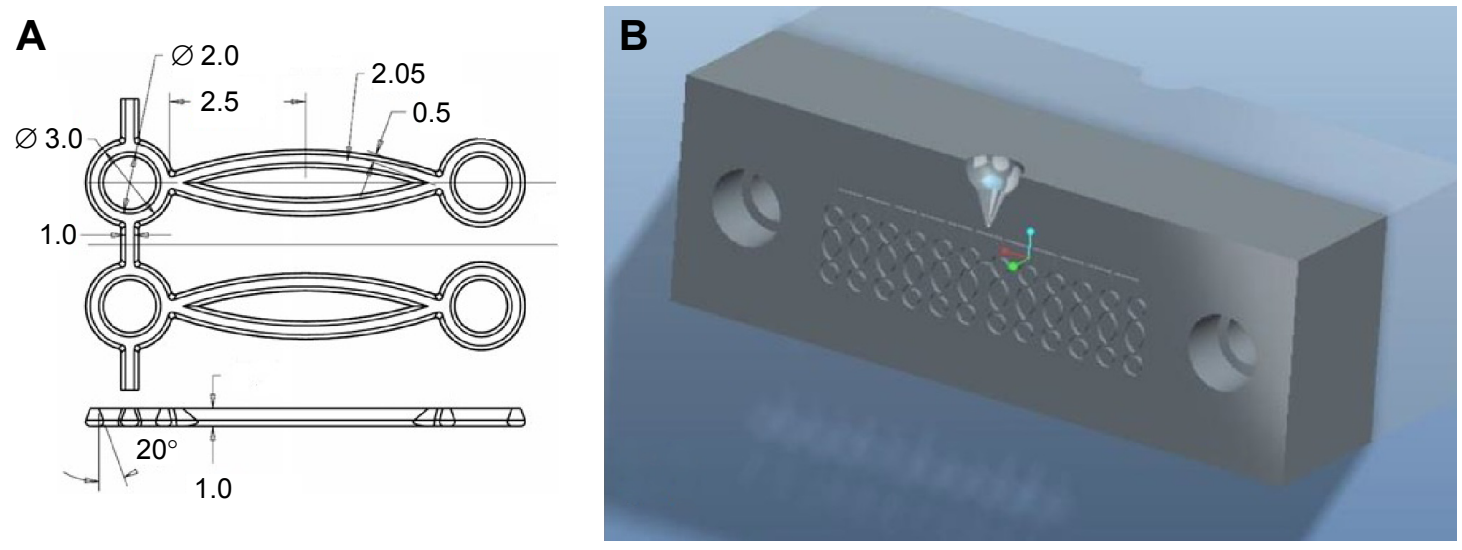

Figure I Stent element and the mold.

Notes: (A) Layout and dimensions of the stent element. (B) The mold used to produce the element. 
interconnected with another element. After interconnecting a total of six elements, the unit was rolled into a mesh membrane (Figure 2).

\section{Preparation of the drug-eluting nanofibrous membrane}

The biodegradable polymer that was employed for the production of the nanofibrous membrane was poly $([\mathrm{D}, \mathrm{L}]-$ lactide-co-glycolide) (PLGA) (Resomer RG 503; Boehringer Ingelheim $\mathrm{GmbH}$, Ingelheim, Germany), which has a lactide-to-glycolide ratio of 50:50 and a molecular weight of 33,000 Da. The drugs used in this study were lidocaine hydrochloride, vancomycin hydrochloride, and ceftazidime hydrate, all of which were obtained from Sigma-Aldrich.

The electrospinning system used to fabricate the nanofibers included a syringe pump and needle, which had an internal diameter of $0.42 \mathrm{~mm}$, an adjustable high-voltage direct current power supply, a ground electrode, and an aluminum collection plate. The needle was connected to the high-voltage power supply that produced a positive direct current voltage and a current of up to $35 \mathrm{kV}$ and $4.16 \mathrm{~mA} / 125 \mathrm{~W}$, respectively. Before the electrospinning procedure began, mixtures containing two different drug-topolymer weight ratios, ${ }^{14}$ namely $1: 3$ and $1: 6$, which comprised $180 \mathrm{mg}$ PLGA, $20 \mathrm{mg}$ lidocaine, $20 \mathrm{mg}$ vancomycin, and $20 \mathrm{mg}$ ceftazidime, and $360 \mathrm{mg}$ PLGA, $20 \mathrm{mg}$ lidocaine, $20 \mathrm{mg}$ vancomycin, and $20 \mathrm{mg}$ ceftazidime, respectively, were dissolved in $1 \mathrm{~mL}$ of 1,1,1,3,3,3-hexafluoro-2-propanol (Sigma-Aldrich). The solutions were then delivered through the syringe pump for electrospinning. The volumetric flow rate of the pump was $0.8 \mathrm{~mL} / \mathrm{h}$. The distance between the needle tip and the collection plate was $9 \mathrm{~cm}$, and the positive voltage applied to the polymer solutions was set at $17 \mathrm{kV}$. The electrospun nanofibers were collected in a non-woven form on the collection plate. The thickness of the electrospun membrane was $0.11 \mathrm{~mm}$. All of the membranes were prepared at room temperature.

After the electrospinning procedure, the morphology of the fibers was examined by coating them with gold and examining them with a scanning electron microscope (SEM) (Hitachi S-3000N; Hitachi Ltd., Tokyo, Japan). The diameter distributions of the fibers were obtained by analyzing the SEM images of 20 randomly selected fibers from each test sample $(n=3)$ using commercial ImageJ image processing software (National Institutes of Health, Bethesda, MD, USA).

\section{In vitro drug release studies}

The in vitro releases of lidocaine, vancomycin, and ceftazidime from the nanofibrous membranes were assessed using the elution method. Samples measuring $1 \times 1 \mathrm{~cm}$ that had been cut from the electrospun membranes were placed individually in glass test tubes $(n=3)$ that contained $1 \mathrm{~mL}$ of phosphate buffer solution (PBS) (0.15 M, pH 7.4). The glass test tubes were incubated at $37^{\circ} \mathrm{C}$ for 24 hours before the membrane eluents were collected and analyzed. Fresh PBS $(1 \mathrm{~mL})$ was then added to the samples, and the tubes were incubated at $37^{\circ} \mathrm{C}$ for 24 hours before the membrane eluents were collected and analyzed, and this procedure was repeated for 35 days. The lidocaine and antibiotic concentrations in the buffer were determined using high-performance liquid chromatography. ${ }^{15}$ The vancomycin activity within the eluent was determined using the disk-diffusion method that was undertaken in nutrient broth that comprised beef extract and
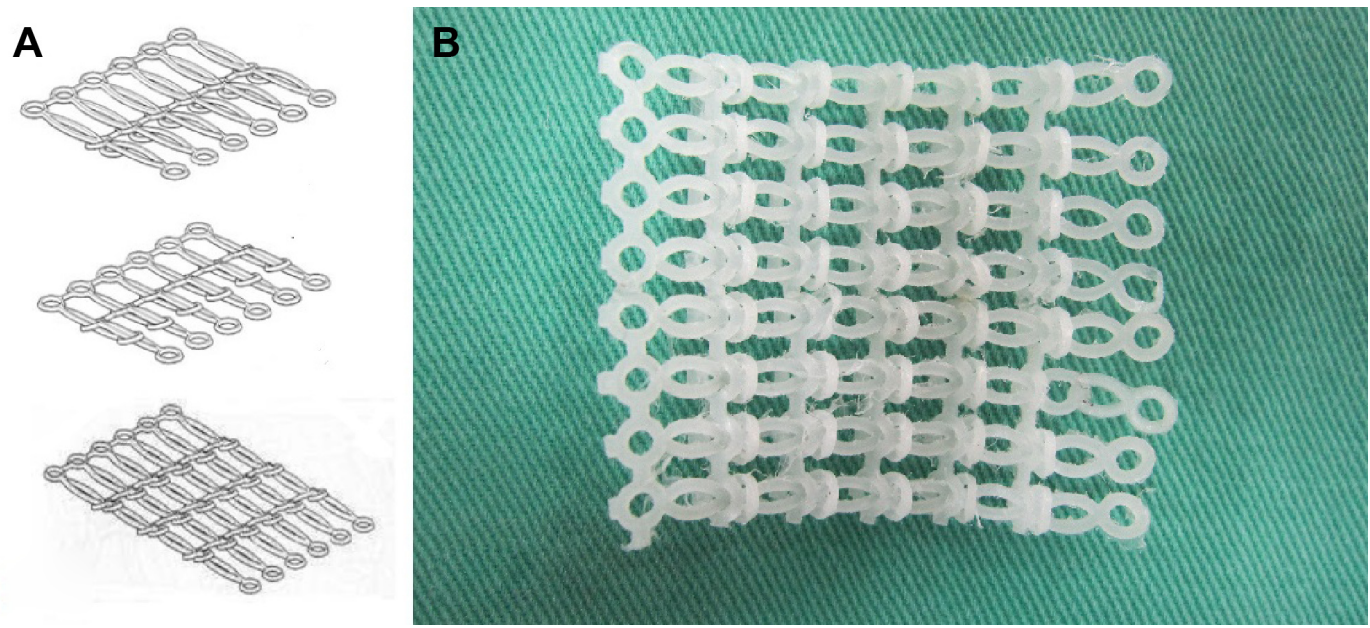

Figure 2 Stent assembly.

Notes: (A) Assembly process of the PCL stent. (B) Assembled stent.

Abbreviation: $\mathrm{PCL}$, poly ( $\varepsilon$-caprolactone). 
peptone (Becton, Dickinson and Company, Franklin Lakes, NJ, USA). The membrane eluent $(8 \mu \mathrm{L})$ was pipetted onto the $6 \mathrm{~mm}$ absorption disks that were placed onto nutrient agar plates that had been seeded with a layer of Staphylococcus aureus (American Type Culture Collection [ATCC] 65389 [Food Industry Research and Development Institute, Hsinchu, Taiwan]). The inhibition zones were measured using a micrometer after incubation for $16-18$ hours at $35^{\circ} \mathrm{C}$. A $50 \mu \mathrm{g} / \mathrm{mL}$ drug solution was used as a reference sample. ${ }^{14}$ The relative activity of the released vancomycin was calculated as follows: relative activity $(\%)=($ diameter of the sample inhibition zone)/(diameter of the reference inhibition zone) $\times 100$. The bioactivity of the ceftazidime on Escherichia coli (ATCC25922) was determined using the same method.

\section{Surgical procedure and animal care}

Twenty-four adult New Zealand white rabbits (Animal Health Research Institute, Panchiao, Taiwan) with a mean weight of $2.9 \pm 0.4 \mathrm{~kg}$ were used in this study. The animal-related procedures in this study were granted institutional approval by the Chang Gung University, and all the animals involved were cared for in a manner that was consistent with the regulations of the National Institute of Health of Taiwan under the supervision of a licensed veterinarian.

General anesthesia was induced in all 24 rabbits through the inhalation of isoflurane (Aesica Queenborough Limited, Queenborough, UK) in an anesthesia chamber that comprised a $40 \times 20 \times 28 \mathrm{~cm}$ transparent acrylic box. Anesthesia was maintained through a mask during the whole surgical procedure. Under sterile conditions, all of the rabbits underwent a longitudinal incision of the left lateral thigh followed by a significant periosteal dissection to expose the left femoral shaft. The first transverse femoral osteotomy was performed $2 \mathrm{~cm}$ proximal to the distal femoral condyle, and the second transverse osteotomy was performed $1 \mathrm{~cm}$ proximal to the first osteotomy site, thereby creating the segmental femoral fracture model (Figure 3A). The surgical wound was extended into the left knee for the lateral parapatellar exposure of the distal femur that enabled a hole to be drilled (Figure 3B) in the intercondylar notch for the insertion of a $2 \mathrm{~mm}$ Kirschner (K)-wire that extended to the proximal
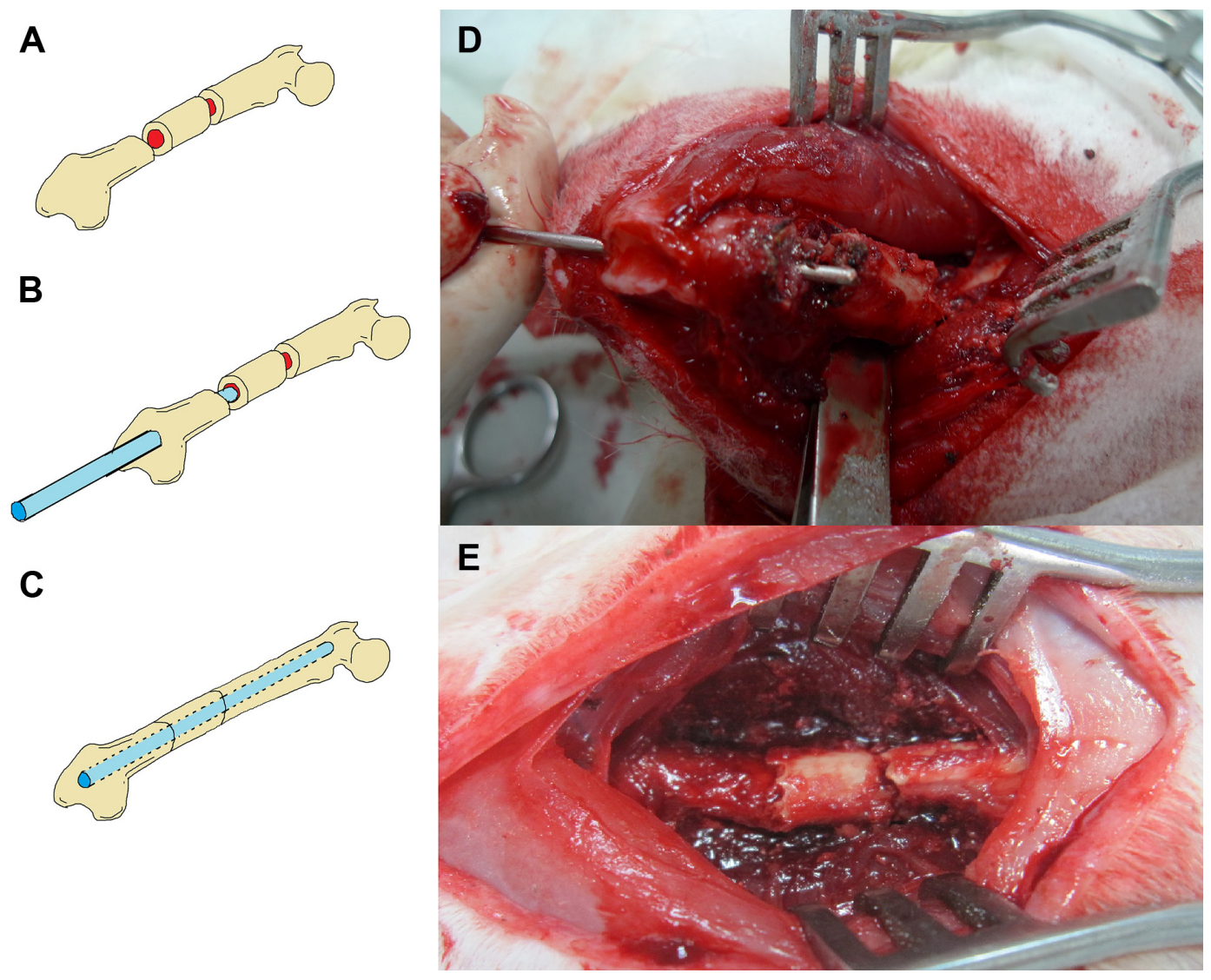

Figure $3 \mathrm{~A}$ rabbit in group $\mathrm{A}$ that received intramedullary K-wire fixation only.

Notes: (A) Illustration shows a segmental fracture on the femoral shaft. (B) Illustration shows an intramedullary K-wire fixation. (C) Illustration demonstrates the final fixation configuration. (D) Photography shows the procedure of the K-wire fixation. (E) Photography demonstrates an acceptable bone alignment after the K-wire fixation. Abbreviation: K-wire, Kirschner-wire. 
end of the femur, thereby creating the intramedullary K-wire fixation (Figure 3C). Figure 3D and E show photographically the surgical procedure. The rabbits were randomly divided into two groups. In group A, 12 rabbits underwent segmental femoral fractures and intramedullary K-wire fixations only, and in group B, 12 rabbits underwent segmental femoral fractures and drug-loaded nanofibrous membranes with polymer-to-drug ratios of 1:6 were inserted into the fracture sites (Figure 4A and B), and they were then enveloped with the PCL stents (Figure 4C). The biodegradable PCL stents were closed and fixed with 3-0 Vicryl ${ }^{\circledR}$ sutures (Johnson \& Johnson, New Brunswick, NJ, USA) of a sufficient tightness to ensure that all of the segmental fracture fragments were covered. Figure 4D and E exhibits photographically the implantation of the stent and nanofibrous membrane.

\section{Postoperative animal care and radiographic assessments}

After surgery, the general activities of all of the rabbits were recorded daily by housing the rabbits in laboratory-made animal behavior cages (ABCs) for 7 days. Then, the rabbits were returned to their original cages for further observations of their activities. As shown in Figure 5, the floor of an ABC measured $1.2 \times 1.2 \mathrm{~m}$, and the cage was $0.7 \mathrm{~m}$ high. On top of the cage, nine diffuse scan-type photoelectric switch sensors with self-contained amplifiers (HP100-A1; Azbil Corp., Chiyoda-ku, Japan) that were separated by a distance of $30 \mathrm{~cm}$ from each other monitored the movements of the rabbits. Before the measurements began, the sensors were adjusted to detect $250 \times 250 \mathrm{~mm}$ objects that moved around the bases of the cages. The nine sensors could detect animal movements within the nine symmetrical areas of the cage. When a rabbit moved from one area into the neighboring area, the sensor in the neighboring area was triggered, and one unit of activity was recorded. The totals of the activity units were recorded using a personal computer equipped with an acquisition interface. All of the animals' activities were monitored for 7 days.

After recording the animals' activities for 1 week, all of the rabbits were allowed to live freely in individual cages, and they were given standard rabbit chow and sterilized drinking water ad libitum. The temperature and humidity
A

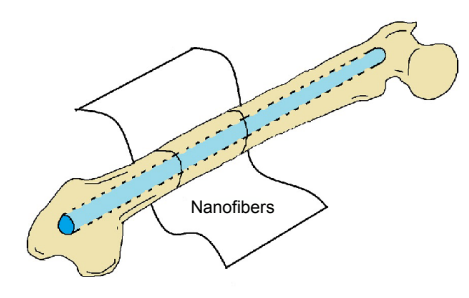

B

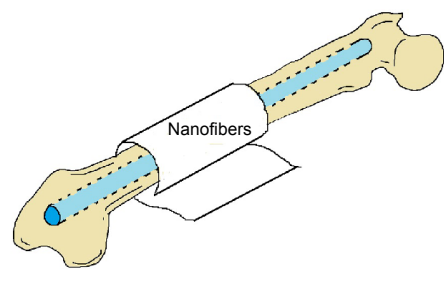

C

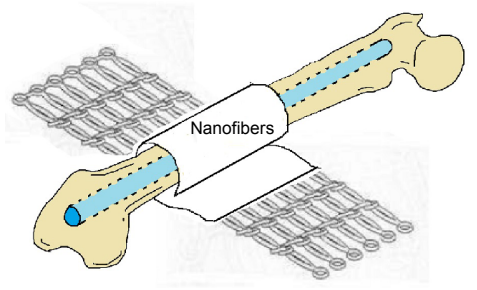

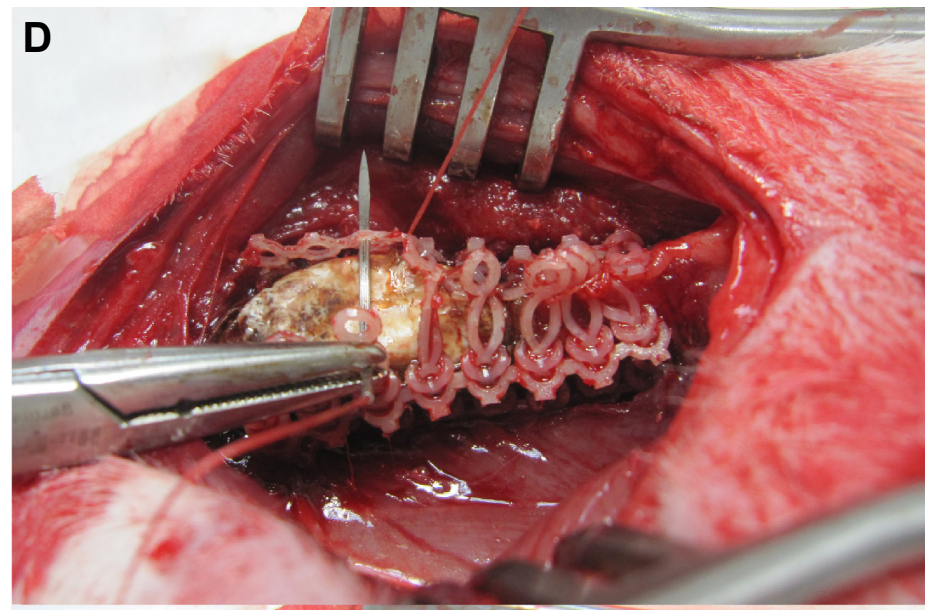

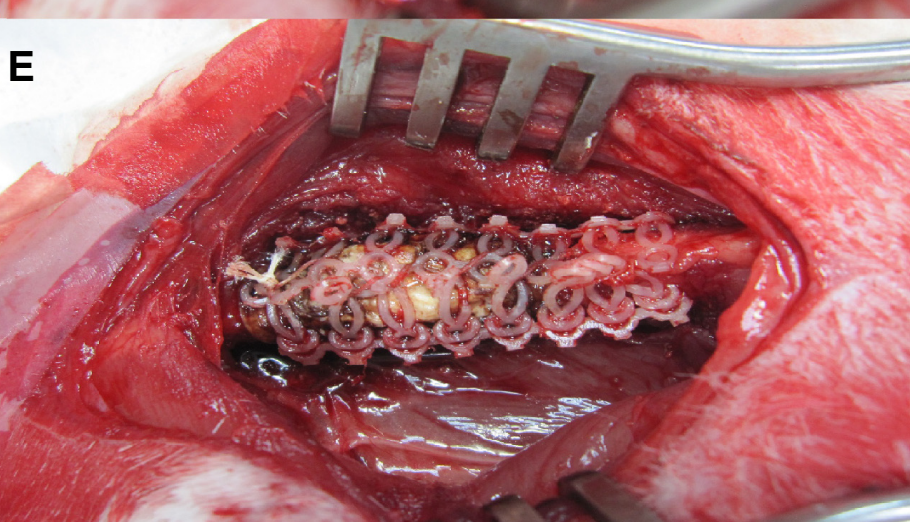

Figure $4 \mathrm{~A}$ rabbit in group $\mathrm{B}$ that received intramedullary K-wire fixation and drug-loaded nanofibrous membrane implantation.

Notes: (A) Illustration shows a nanofibrous membrane prepared around the fracture site. (B) Illustration shows the nanofibrous membrane enfolded on the fracture site. (C) Illustration shows the PCL stents wrapped around the fracture site. (D) Photography shows the procedure of suturing the PCL stents. (E) Photography demonstrates the final composite nanofibrous membrane and PCL stents around the fracture site.

Abbreviation: PCL, poly ( $\varepsilon$-caprolactone). 

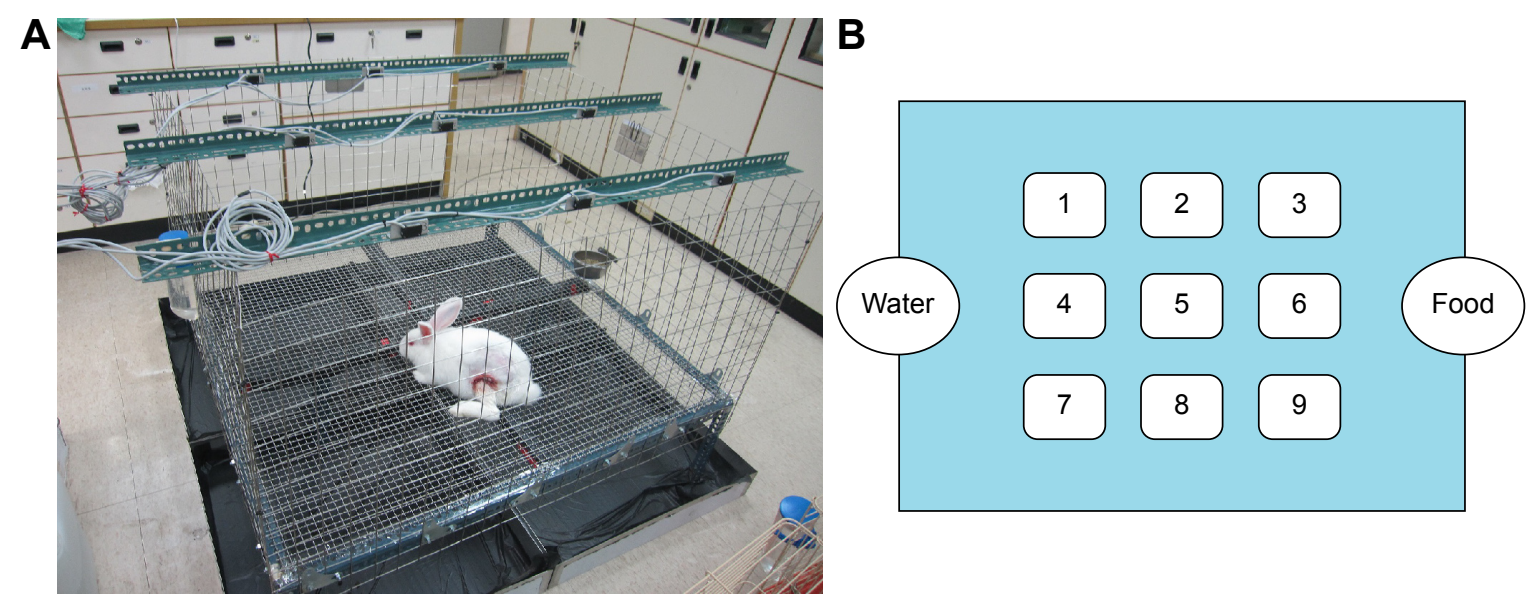

Figure 5 The device for animal activity test.

Notes: (A) The animal bioactivity cage. (B) The layout of the cage. Each number in the cage represents the sensor number.

were maintained at $24^{\circ} \mathrm{C}$ and $70 \%$, respectively. All of the rabbits underwent radiographic assessments of their left thighs at 2, 6, and 12 weeks postoperatively to evaluate the bone-healing process.

\section{Animal euthanasia and mechanical strength tests}

After completing the final imaging studies at 12 weeks postoperatively, all of the rabbits were euthanized by intravenous injections of lidocaine, and the mechanical strength of the bones was tested. Both the left study femurs and the right healthy femurs were harvested from the rabbits for the clinical comparisons. The femoral specimens were dissected to remove the residual muscles and to gently remove the intramedullary K-wire. Both ends of each femoral specimen were stabilized by embedding the femoral bones in epoxy blocks that were cylindrically shaped, had $1.5 \mathrm{~cm}$ radii, and were $1.3 \mathrm{~cm}$ thick. The specimens were then secured onto a mechanical strength machine (Gotech Testing Machines, Inc., Taichung, Taiwan) (Figure 6A) for the rotational torque and toughness assessments (Figure 6B).

\section{Statistical analysis}

The data collected from the samples were analyzed using SPSS software, version 12.0 (SPSS Inc., Chicago, IL, USA). A least significant difference test was used to compare the two groups. A chi-square test was used for the nonparametric data, namely the activity performance scores. The data are expressed as the means and the standard deviations, and a $P$-value of $<0.05$ was considered statistically significant.

\section{Results}

The drug-eluting nanofibrous membrane was successfully fabricated using the electrospinning technique. Figure 7

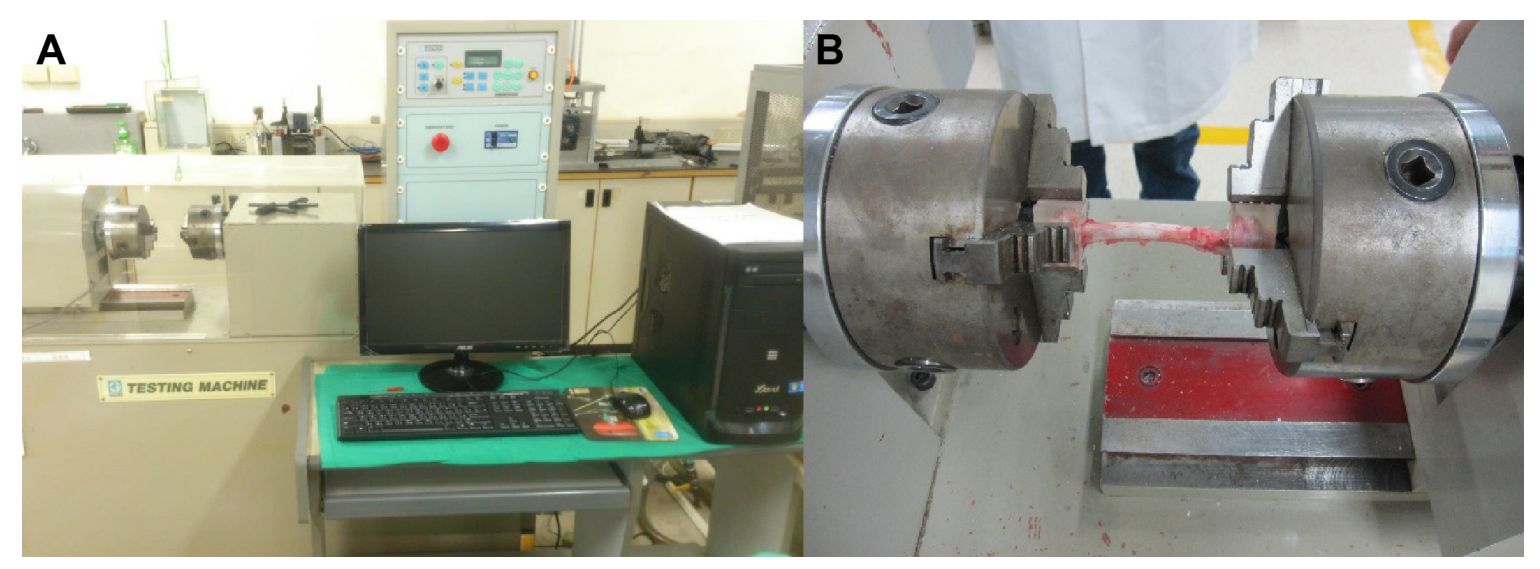

Figure 6 Torsional test of bone specimens.

Notes: (A) Photograph of the torsional testing machine. (B) Bone specimens were secured onto a mechanical strength machine for the rotational torque and toughness assessments. 

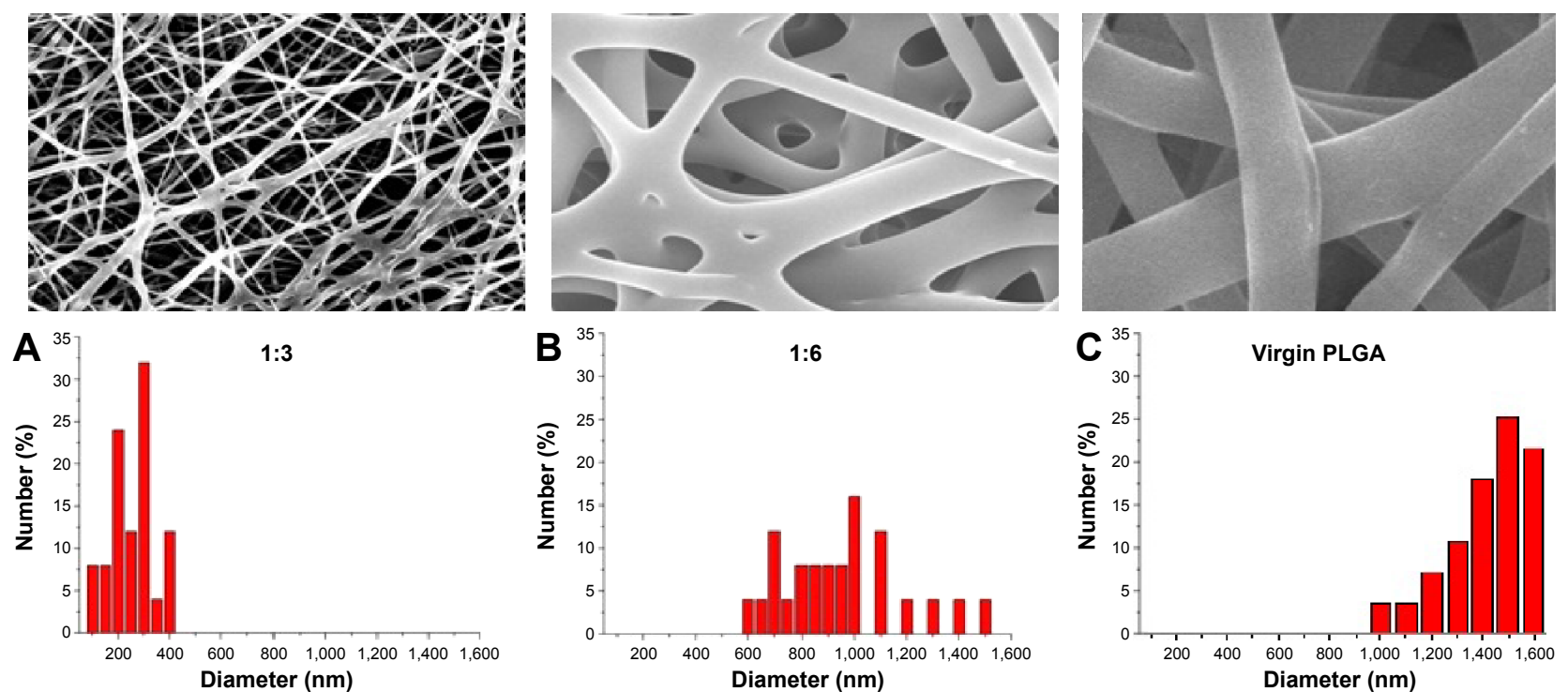

Figure 7 SEM images and fiber diameter distributions of electrospun PLGA/lidocaine nanofibers.

Notes: The average diameters of the nanofibers of drug-to-polymer ratios were (A) $280 \pm 126 \mathrm{~nm}$ in the I:3 ratio, (B) $980 \pm 247 \mathrm{~nm}$ in the I:6 ratio, and (C) I405 \pm I68 $\mathrm{nm}$ in the virgin nanofibers (without drug loading). $\times 2,000$ Magnification.

Abbreviations: SEM, scanning electron microscope; PLGA, poly([D,L]-lactide-co-glycolide).

shows the SEM images of the nanofibers at a magnification of $\times 20,000$. All of the electrospun nanofibers showed high levels of porosity. The average diameter of the nanofibers from the mixture that contained the 1:3 and 1:6 drug-topolymer ratios was $280 \pm 126$ and $980 \pm 247 \mathrm{~nm}$, respectively, while the average diameter of virgin nanofibers (without drug loading) was $1,405 \pm 168 \mathrm{~nm}$.

\section{In vitro release characteristics of lidocaine from the nanofibers}

The daily and accumulated releases of lidocaine from the nanofibrous membranes are shown in Figure 8A and B, respectively. Lidocaine exhibited a triphasic release pattern, with an initial burst release on day 1 that was followed by a decline in the release until day 5. A second drug release peak was observed from days 13 to 15 , after which the drug release from the nanofibers diminished progressively over $>24$ days for both of the drug-to-polymer ratios.

\section{In vitro antibiotic releases}

Figure 9A and B show the daily and accumulated release curves for vancomycin, while Figure 10A and B display the daily and accumulated release curves for ceftazidime. The influence of the drug-to-polymer ratio on the in vitro release characteristics of the antibiotics from the nanofibrous membranes was investigated. The nanofibrous membranes containing the different drug concentrations exhibited similar antibiotic release patterns, which were characterized by high
A

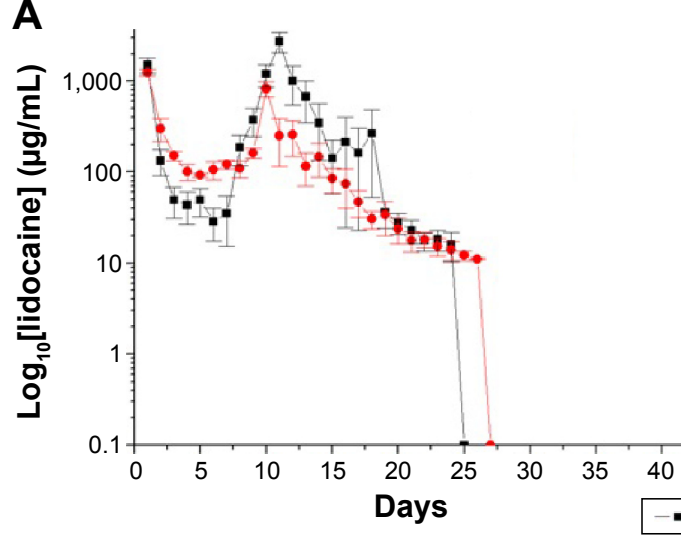

B

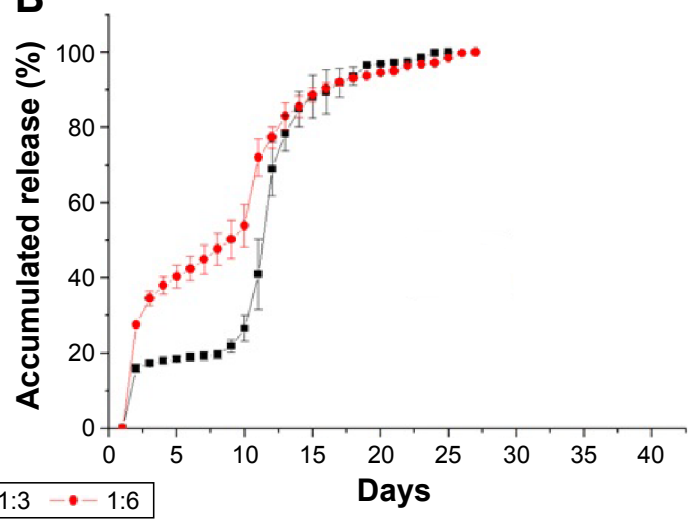

Figure 8 In vitro release curves of lidocaine.

Notes: (A) Daily and (B) accumulated releases of lidocaine from the nanofibrous membranes (drug:polymer). I:3=180 mg PLGA, 20 mg lidocaine, 20 mg vancomycin, and $20 \mathrm{mg}$ ceftazidime. I:6=360 mg PLGA, $20 \mathrm{mg}$ lidocaine, $20 \mathrm{mg}$ vancomycin, and $20 \mathrm{mg}$ ceftazidime. 

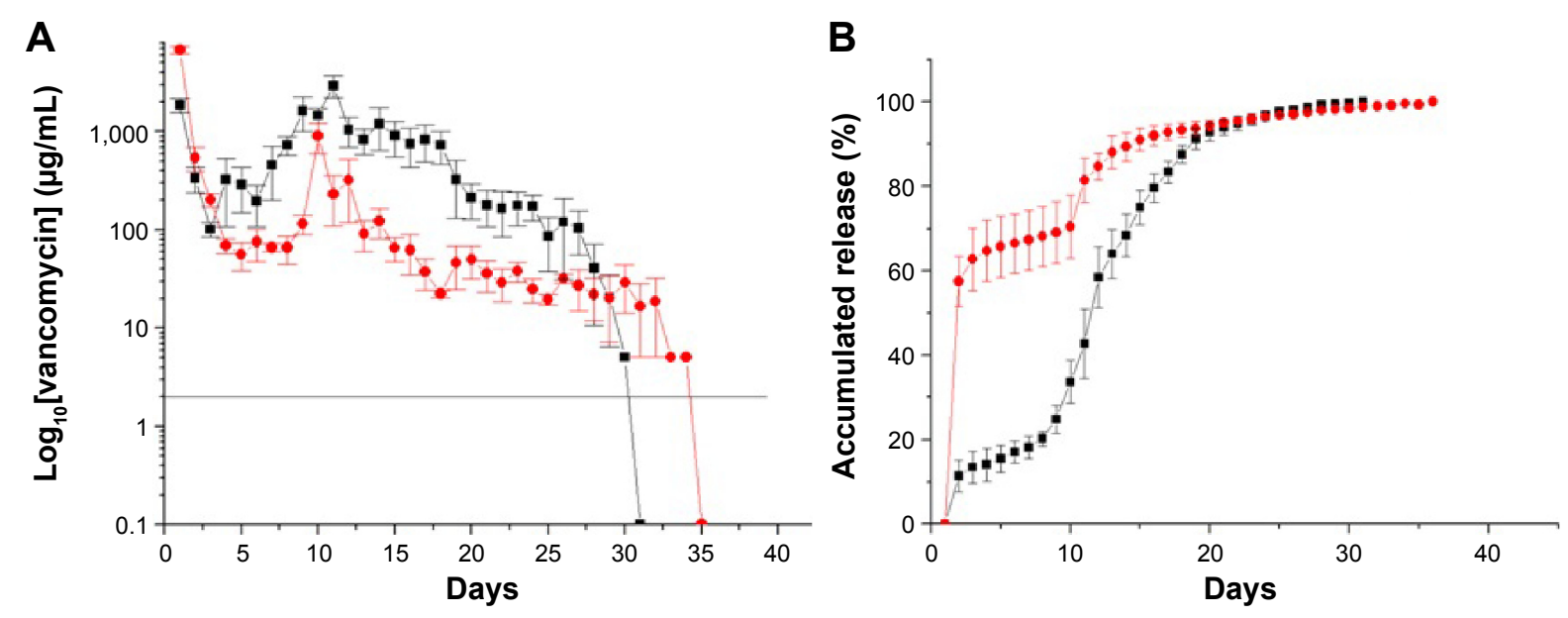

$-1: 3-1: 6$

Figure 9 In vitro release curves of vancomycin.

Notes: (A) Daily and (B) accumulated release curves for vancomycin (drug:polymer). I:3=180 mg PLGA, 20 mg lidocaine, 20 mg vancomycin, and 20 mg ceftazidime. I:6=360 mg PLGA, $20 \mathrm{mg}$ lidocaine, $20 \mathrm{mg}$ vancomycin, and $20 \mathrm{mg}$ ceftazidime.

releases during the first 2 days, and major release peaks at 10 days, followed by gradual and sustained drug releases. The membranes containing the different drug-to-polymer ratios released high concentrations of the antibiotics that were well above the minimum inhibitory concentrations for $>30$ days. The in vitro release of ceftazidime was more stable, with only minor initial burst releases observed. The bioactivities of the eluted vancomycin on $S$. aureus and of the eluted ceftazidime on $E$. coli were determined using an antibiotic disk-diffusion method. Figure 11A and B show the bioactivities of the released vancomycin and ceftazidime, respectively. The antibiotics' bioactivities remained high after the electrospinning process.
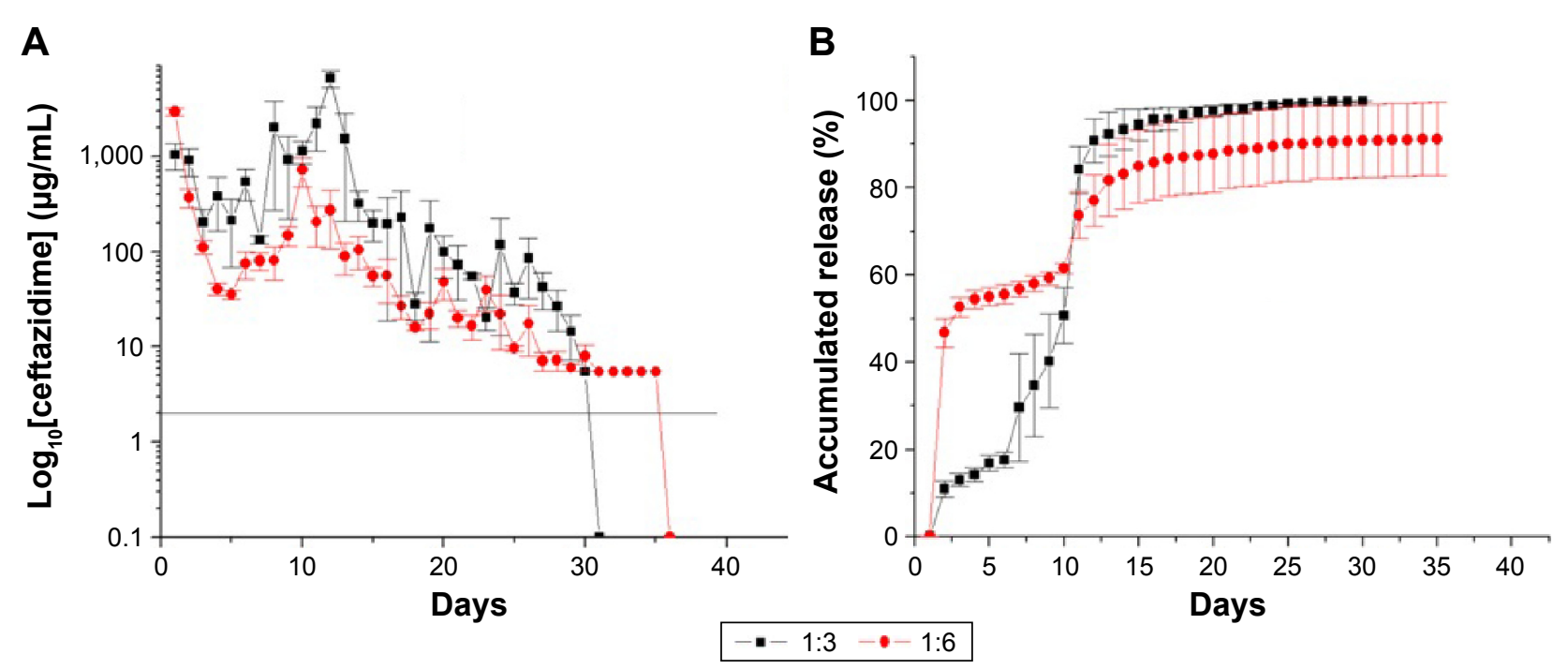

\section{In vivo animal activity results}

All 24 rabbits completed the 12-week postoperative care course, and there were no deaths. None of the rabbits in either group developed deep wound infections, hematomas, or seromas around the fracture sites. Two rabbits in group B and one rabbit in group A demonstrated wound dehiscence because the rabbits had bitten their wounds. The ruptured wounds were treated with topically applied antibiotic ointment and no sutures were required, and they healed progressively and formed scars.

The mean activity counts at 1 week postoperatively, which were based on the recordings from the photoelectric switch sensors placed on the ABCs, were 4,484 \pm 366

Figure 10 In vitro release curves of ceftazidime.

Notes: (A) Daily and (B) accumulated release curves for ceftazidime (drug:polymer). I:3=180 mg PLGA, 20 mg lidocaine, 20 mg vancomycin, and 20 mg ceftazidime. I:6=360 mg PLGA, $20 \mathrm{mg}$ lidocaine, $20 \mathrm{mg}$ vancomycin, and $20 \mathrm{mg}$ ceftazidime. 
A

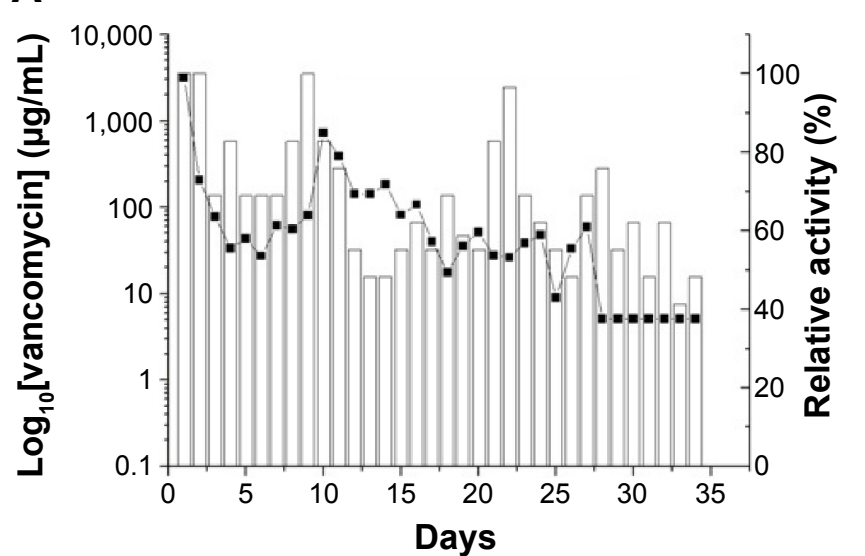

B

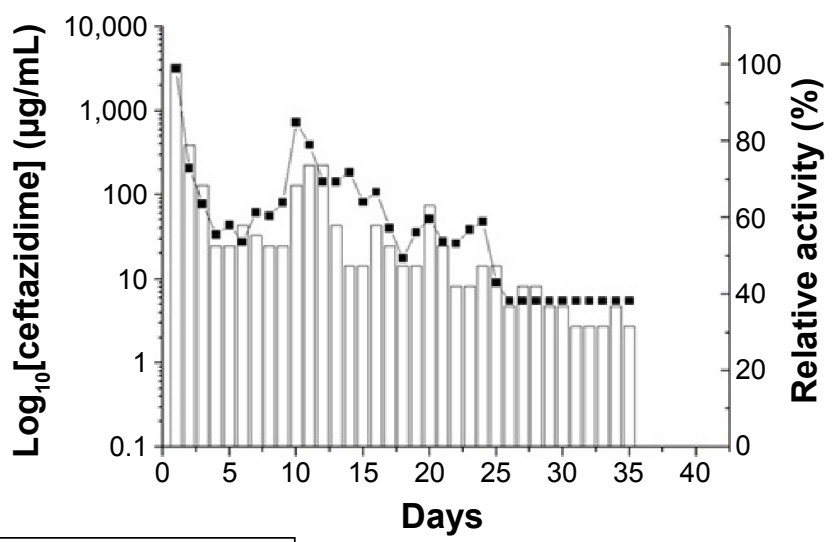

$-1: 6$ concentration $\square$ 1:6 bioactivity

Figure I I Bioactivities of antibiotics.

Notes: Bioactivities of eluted (A) vancomycin and (B) ceftazidime at various days. I:6=360 mg PLGA, 20 mg lidocaine, 20 mg vancomycin, and 20 mg ceftazidime.

and $6,856 \pm 716$ counts for groups A and B, respectively (Figure 12). Group B showed a higher number of sensor triggers than group $\mathrm{A}$, indicating that the animals that had received the biodegradable nanofiber and PCL stent treatment were more active.

\section{Radiographic bone healing in vivo}

The radiographic assessments at 2 weeks postoperatively showed that the intramedullary K-wire had migrated in three rabbits $(25 \%, 3 / 12)$ in group A and in two rabbits $(16.6 \%$, $2 / 12$ ) in group B, and that the K-wires partially bulged out from the femoral intercondylar insertion site. The radiographs at 6 weeks postoperatively showed that the K-wire was partially bulging out from the insertion site in five rabbits in group A and in two rabbits in group B. The radiographic assessments at 12 weeks postoperatively showed delayed bone unions in five rabbits in group A and in one rabbit in

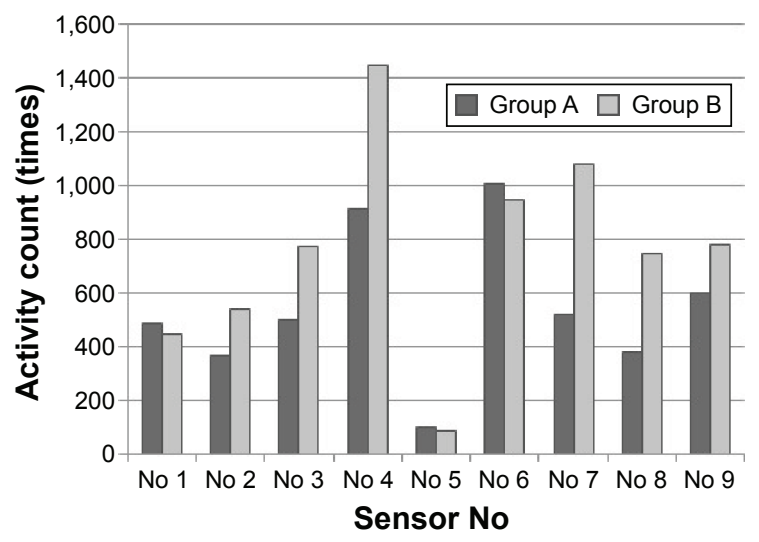

Figure 12 The mean activity counts at I week postoperatively. group B, and the formation of the bridging calluses was inadequate within the gaps produced by the fractures. The clinical bone union rates at 12 weeks postoperatively were $58.3 \%(7 / 12)$ in group A and $91.6 \%(11 / 12)$ in group B. A statistically significant difference in the bone-healing rate was noted between the groups at the 12-week radiographic assessment (Figure 13A1-A3 and B1-B3).

\section{In vivo mechanical strength}

The mechanical strength tests excluded those rabbits from both groups that showed delayed bone unions; hence, seven rabbits from group A and eleven rabbits from group B were included in the strength tests. To diminish the bias associated with the different bone qualities among the individual rabbits, the maximum torque of the left study femur was divided by that of the right healthy femur to obtain the torque strength ratio. The same method was used to obtain the toughness ratios from all of the rabbits. Group A's torque strength ratio (73.3\%) was lower than that of group B (81.6\%), a difference that was not statistically significant $(P>0.05)$. The toughness ratio was significantly lower in group A (63.3\%) compared with group B $(91.6 \%)(P<0.05)$.

\section{Discussion}

The treatment of AO type IIIB open fractures that have significant soft tissue damage always requires staged surgical procedures. An initial damage-control operation is recommended to remove all contaminating materials and to provide temporary bone stabilization. ${ }^{2,16}$ All devitalized skin, muscle, periosteum, and sequestrated bone tissue should be excised during debridement procedures to gain sufficient control of 

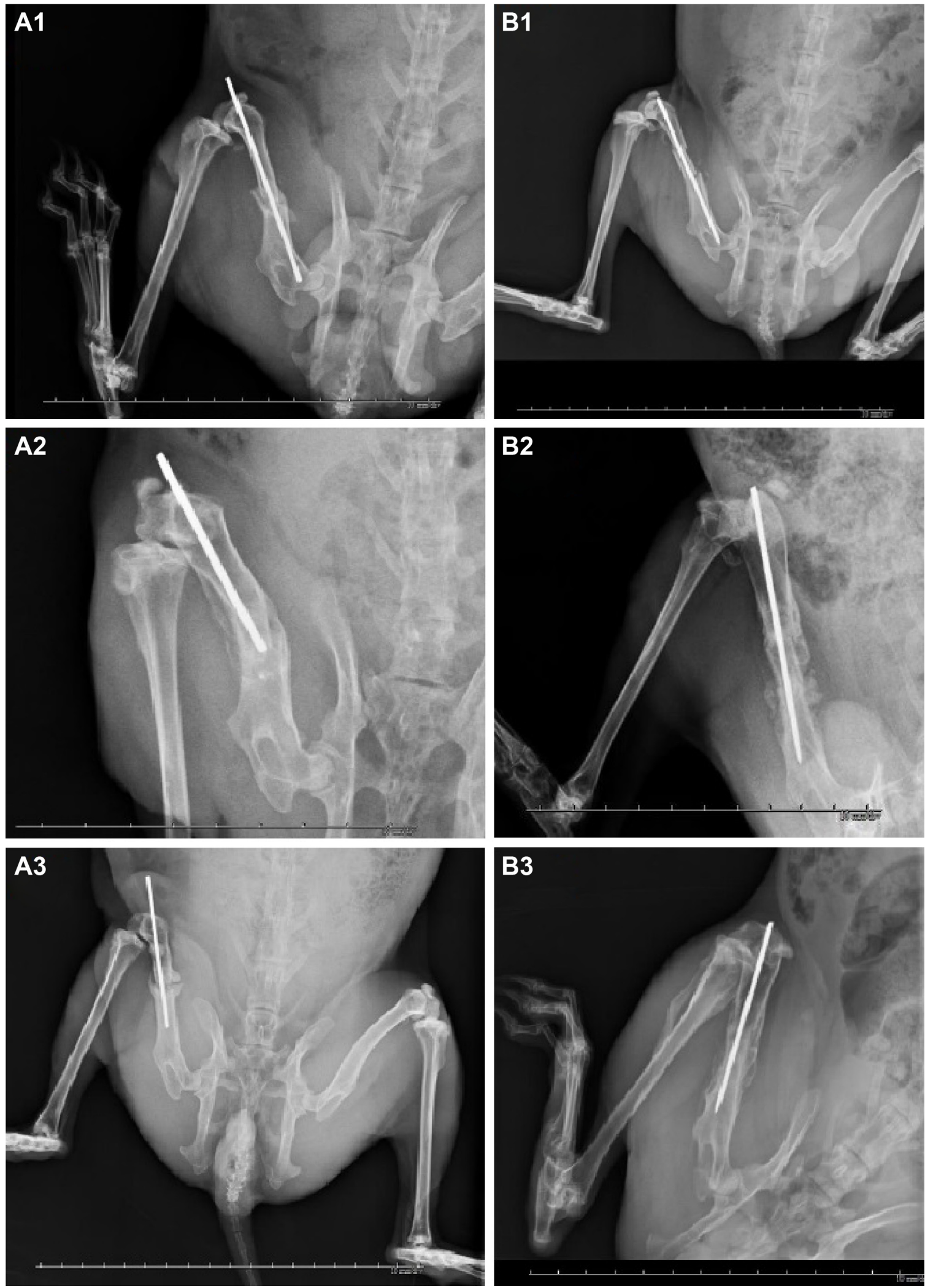

Figure 13 Radiographic assessments of rabbits' left thighs.

Notes: (AI) A rabbit in group A showed breakage of the middle femoral segment at 2-week follow-up. (A2) Some callus formation and migration of the K-wire were noted at 6-week follow-up. (A3) Nonunion of the femoral shaft and loosening of the K-wire were diagnosed at I2-week follow-up. (BI) A rabbit in group B showed adequate initial fracture fixation at 2-week follow-up. (B2) Partial callus formation was noted around the fracture site at 6-week follow-up. (B3) An adequate bone healing was noted at I2-week follow-up. Each frame rates one centimeter.

Abbreviation: K-wire, Kirschner-wire. 
infections. However, during further steps of surgical procedures, few surgeons pay sufficient attention to the restoration of the periosteum, which contains osteogenic progenitor cells, provides a source of osteoinductive growth factors, and provides an osteoconductive structure, ${ }^{8,9,17}$ and is, therefore, a critical component of the bone-healing process. In this study, we developed a biodegradable drug-eluting PLGA nanofibrous membrane that was combined with PCL stents of sufficient elastic strength to mimic the periosteum.

The results from the in vitro drug release experiments indicated that the nanofibers can provide effective concentrations of vancomycin and ceftazidime for longer than 30 days, which would promote infection control; hence, the nanofibrous membrane may be an ideal drug-eluting system that enables the sustained release of antibiotics. The results from the animal study, which used a rabbit femoral segmental fracture model, showed that the artificial drug-eluting periosteum provided adequate biomechanical compatibility without inducing tissue overreactions. The sustained release of lidocaine from the nanofibers encouraged the rabbits to be more active during the first week postoperatively. The good elastic strength of the PCL stents that formed a circumferential envelope around the segmental fracture site also enhanced the mechanical toughness of the femoral shaft.

Tseng et al reported the development of lidocaineembedded PLGA nanofibers for the sustained delivery of lidocaine into the epidural space in rats to provide pain relief after laminectomy. ${ }^{18}$ Vancomycin-eluting PLGA nanofibers have been applied to the treatment of brain abscesses, and vancomycin/ceftazidime-eluting nanofibers have been applied to plates and screws to treat osteomyelitis. ${ }^{19,20}$ It is widely accepted that carriers for local antibiotic delivery can provide sustained releases of high concentrations of antibiotics to sites of infection. Previous studies have reported that vancomycin is effective when it is used in polymethylmethacrylate cement beads to control infections. ${ }^{21}$ However, the nondegradable nature of the polymethylmethacrylate drug delivery system limits its clinical application. The development of a biodegradable carrier is considered theoretically advantageous because of the potential reduction in the risk of secondary infections and the elimination of the need for further surgery to remove the implant. ${ }^{22}$

Drugs formulated in biodegradable polymeric vehicles are released by diffusion through the polymer barrier, or by erosion of the polymer material, or by a combination of both diffusion and erosion mechanisms. With a degradation time of $\sim 2$ months, PLGAs have been widely employed for the delivery of various pharmaceuticals. With respect to the nanofibers that were embedded with lidocaine and antibiotics in this study, we found that the drug release behavior varied in association with the different drug-to-polymer ratios. Since all drug-eluting nanofibers possessed sustained drug release capacities that spanned $>3$ weeks, we recommend that the nanofibers that incorporated the lower drug-topolymer ratio (1:6) are used in future applications. This is because the nanofibers with the higher drug-to-polymer ratio (1:3) showed greater peak burst releases of lidocaine and vancomycin during days 1 and 2 postoperatively, which could increase the risk of local or systemic tissue damage caused by drug overdoses during the burst release periods.

Many drugs degrade slowly and are highly permeable, and PCL was initially investigated as a long-term drug or vaccine delivery vehicle. The semi-crystalline nature and significant elasticity at room temperature have rendered PCL unsuitable for fracture fixation implants in the orthopedic field. ${ }^{23-25}$ However, many publications have described the experimental application of PCL as a scaffold to fill bone gaps and to provide osteoconduction in bone engineering. ${ }^{26}$ We expanded the experimental application of PCL in this study in which it was used as a circumferential scaffold around the fracture site that mimicked the periosteum and provided elastic strength for fracture fragment fixation. By enveloping them in the PCL stent, the nanofibers could adhere to the segmental fracture site more effectively without shedding migration or self-contracture within the body fluid. Roberts ${ }^{27}$ first proposed the concept of artificial periosteum for fixation of shaft fractures. Hattori et $\mathrm{al}^{28}$ developed a bio-artificial periosteum composed of osteogenic cells and collagen sponge. By using a rat model for severe bone injury where the defect sites were covered with the bio-artificial periosteum, their results demonstrated new bone formation in the central part of the bone defect as well as at the bone edge. Shi et $\mathrm{al}^{29}$ developed microgrooved PLGA nanosheets that adhered on the surfaces of bones before and after incubation in PBS for 14 days. Compared to the previous bio-artificial periosteum that contained live cells, the PLGA artificial periosteum/PCL stent developed in this study consisted only of biodegradable nanofibers and commonly used drugs. The porous structure of the nanofibrous mat can mimic the structure of extracellular matrix and promote tissue/bone regeneration, while the PCL stent provides mechanical support for the nanofibrous membrane to stick tightly to the bones. This provides advantages in terms of easy manufacture of the artificial periosteum, high mechanical support of the periosteum by the biodegradable stent, as well as 
good cell proliferation and extracellular matrix deposition with subsequent ingrowth until the tissues/bones are totally restored or regenerated.

Despite the proven effectiveness of the drug-eluting nanofibers and the elastic strength of the PCL stents that enabled the imitation of the periosteum, there were some limitations associated with this study. The first shortcoming was that we could not design an open-fracture animal model that had significant amounts of damage to the periosteum and soft tissue defects that required flap reconstruction because the prolonged surgery and the associated animal care following the microsurgery would have increased the risk of rabbit death. Hence, we were only able to create a segmental fracture with significant periosteal stripping that simulated the compromise of the periosteum in the segmental femoral fracture model. The second study shortcoming was associated with not performing a bacterial seeding procedure during the in vivo study to mimic the wound contamination that occurs during open fractures. We evaluated the bactericidal capacities of the antibiotics that were eluted from the nanofibers using in vitro high-performance liquid chromatography assessments and antibiotic-specific disk-diffusion assays. ${ }^{17,18}$ The third study limitation relates to this being a preliminary study of the development of an artificial periosteum, and consequently, we did not add any osteogenic progenitors or osteoinductive growth factors to the nanofibers.

To substantiate the feasibility of the use of this drugeluting artificial periosteum in the treatment of open fractures, our future work will involve modifying our animal fracture model to create femoral shaft open fractures with soft tissue defects and inoculating the wound with bacteria to mimic complex open fractures with soft tissue compromise and wound contamination. Furthermore, to expand the capacity of this biodegradable artificial periosteum, particularly its capacity for promoting bone healing, we should also incorporate effective osteoinductive growth factors, including collagen and bone marrow proteins, into the drug-eluting nanofibers to promote bone healing during the treatment of open fractures.

\section{Conclusion}

In this study, we successfully developed an artificial drugeluting periosteum for segmental femoral fractures using biodegradable drug-loaded nanofibers and stents. With its sustained drug-eluting capacity that occurs over a sufficient period of time, the artificial periosteum developed can provide satisfactory pain relief, reasonable biomechanical strength, and a tolerable bactericidal capacity for the management of complex open fractures.

\section{Acknowledgments}

The authors thank Dr Jau-Wen Huang and the staff of the $\mathrm{Ci}-\mathrm{Wen}$ Clinic for their assistance with preparing the images and radiographs for this study. The authors would also like to thank the Ministry of Science and Technology, Taiwan (contract no MOST 104-2221-E-182-048-MY3) and Chang Gung Memorial Hospital (contract no CMRPD3D0151) for supporting this research financially.

\section{Disclosure}

The authors report no conflicts of interest in this work.

\section{References}

1. Hah YS, Joo HH, Kang YH, et al. Cultured human periosteal-derived cells have inducible adipogenic activity and can also differentiate into osteoblasts in a perioxisome proliferator-activated receptor-mediated fashion. Int J Med Sci. 2014;11(11):1116-1128.

2. van Gastel N, Stegen S, Stockmans I, et al. Expansion of murine periosteal progenitor cells with fibroblast growth factor 2 reveals an intrinsic endochondral ossification program mediated by bone morphogenetic protein 2. Stem Cells. 2014;32(9):2407-2418.

3. Oktaş B, Orhan Z, Erbil B, Değirmenci E, Ustündağ N. Effect of extracorporeal shock wave therapy on fracture healing in rat femural fractures with intact and excised periosteum. Eklem Hastalik Cerrahisi. 2014;25(3):158-162.

4. Gustilo RB, Mendoza RM, Williams DN. Problems in the management of type III (severe) open fractures: a new classification of type III open fractures. J Trauma. 1984;24(8):742-746.

5. Malhotra AK, Goldberg S, Graham J, et al. Open extremity fractures: impact of delay in operative debridement and irrigation. J Trauma Acute Care Surg. 2014;76(5):1201-1207.

6. Gopal S, Majumder S, Batchelor AGB, Knight SL, De Boer P, Smith RM. Fix and flap: the radical orthopedic and plastic treatment of severe open fractures of the tibia. J Bone Joint Surg Br. 2000;82(7): 959-966.

7. Harley BJ, Beaupre LA, Jones CA, Dulai SK, Weber DW. The effect of time to definitive treatment on the rate of nonunion and infection in open fractures. J Orthop Trauma. 2002;16(7):484-490.

8. Demirtas Y, Kelahmetoglu O, Cifci M, Tayfur V, Demir A, Guneren E. Comparison of free anterolateral thigh flaps and free muscle-musculocutaneous flaps in soft tissue reconstruction of lower extremity. Microsurgery. 2010;30(1):24-31.

9. Tielinen L, Lindahl JE, Tukiainen EJ. Acute unreamed intramedullary nailing and soft tissue reconstruction with muscle flaps for the treatment of severe open tibial shaft fractures. Injury. 2007;38(8):906-912.

10. Hoffman MD, Xie C, Zhang X, Benoit DS. The effect of mesenchymal stem cells delivered via hydrogel-based tissue engineered periosteum on bone allograft healing. Biomaterials. 2013;34(35):8887-8898.

11. Almodóvar J, Mower J, Banerjee A, Sarkar AK, Ehrhart NP, Kipper MJ. Chitosan-heparin polyelectrolyte multilayers on cortical bone: periosteum-mimetic, cytophilic, antibacterial coatings. Biotechnol Bioeng. 2013;110(2):609-618. 
12. Zhang X, Awad HA, O'Keefe RJ, Guldberg RE, Schwarz EM. A perspective: engineering periosteum for structural bone graft healing. Clin Orthop Relat Res. 2008;466(8):1777-1787.

13. Liu SJ, Chiang FJ, Hsiao CY, Kau YC, Liu KS. Fabrication of balloonexpandable self-lock drug-eluting polycaprolactone stents using microinjection molding and spray coating techniques. Ann Biomed Eng. 2010;38(10):3185-3194.

14. Chen DW, Liao JY, Liu SJ, Chan EC. Novel biodegradable sandwichstructured nanofibrous drug-eluting membranes for repair of infected wounds: an in vitro and in vivo study. Int J Nanomedicine. 2012;7: 763-771.

15. Crandon JL, MacVane SH, Nicolau DP. Clinical laboratory-based assay methodologies may underestimate and increase variability of vancomycin protein binding in hospitalized patients. Pharmacotherapy 2014;34(2):203-209.

16. Mooney JF. The use of 'damage control orthopedics' techniques in children with segmental open femur fractures. JPediatr Orthop B. 2012; 21(5):400-403.

17. Ichikawa Y, Watahiki J, Nampo T, et al. Differences in the developmental origins of the periosteum may influence bone healing. J Periodontal Res. 2015;50(4):468-478.

18. Tseng YY, Liao JY, Chen WA, Kao YC, Liu SJ. Biodegradable poly([D,L]-lactide-co-glycolide) nanofibers for the sustainable delivery of lidocaine into the epidural space after laminectomy. Nanomedicine (Lond). 2014;9(1):77-87.

19. Tseng YY, Kao YC, Liao JY, Chen WA, Liu SJ. Biodegradable drug-eluting poly[lactic-co-glycol acid] nanofibers for the sustainable delivery of vancomycin to brain tissue: in vitro and in vivo studies. ACS Chem Neurosci. 2013;4(9):1314-1321.

20. Hsu YH, Chen DW, Tai CD, et al. Biodegradable drug-eluting nanofiber-enveloped implants for sustained release of high bactericidal concentrations of vancomycin and ceftazidime: in vitro and in vivo studies. Int J Nanomedicine. 2014;9:4347-4355.
21. Donegan DJ, Scolaro J, Matuszewski PE, Mehta S. Staged bone grafting following placement of an antibiotic spacer block for the management of segmental long bone defects. Orthopedics. 2011;34(11):e730-e735.

22. Ferguson JY, Dudareva M, Riley ND, Stubbs D, Atkins BL, McNally MA. The use of a biodegradable antibiotic-loaded calcium sulphate carrier containing tobramycin for the treatment of chronic osteomyelitis: a series of 195 cases. Bone Joint J. 2014;96-B(6): 829-836.

23. Woodruff MA, Hutmacher DW. The return of a forgotten polymer - polycaprolactone in the 21 st century. Prog Polym Sci. 2010; 35:1217-1256

24. Pfeiffer D, Stefanitsch C, Wankhammer K, et al. Endothelialization of electrospun polycaprolactone (PCL) small caliber vascular grafts spun from different polymer blends. J Biomed Mater Res A. 2014;102(12): 4500-4509.

25. Chao YK, Liu KS, Wang YC, Huang YL, Liu SJ. Biodegradable cisplatin-eluting tracheal stent for malignant airway obstruction: in vivo and in vitro studies. Chest. 2013;144(1):193-199.

26. Thadavirul N, Pavasant P, Supaphol P. Improvement of dual-leached polycaprolactone porous scaffolds by incorporating with hydroxyapatite for bone tissue regeneration. J Biomater Sci Polym Ed. 2014; 25(17):1986-2008.

27. Roberts JB. The artificial periosteum for fixation of shaft fractures. Ann Surg. 1916;63(2):182-184.

28. Hattori K, Yoshikawa T, Takakura Y, Aoki H, Sonobe M, Tomita N. Bio-artificial periosteum for severe open fracture - an experimental study of osteogenic cell/collagen sponge composite as a bio-artificial periosteum. Biomed Mater Eng. 2005;15(3):127-136.

29. Shi X, Fujie T, Saito A, et al. Periosteum-mimetic structures made from freestanding microgrooved nanosheet. Adv Mater. 2014;26: 3290-3296.
International Journal of Nanomedicine

\section{Publish your work in this journal}

The International Journal of Nanomedicine is an international, peerreviewed journal focusing on the application of nanotechnology in diagnostics, therapeutics, and drug delivery systems throughou the biomedical field. This journal is indexed on PubMed Central, MedLine, CAS, SciSearch $\AA$, Current Contents $₫ /$ Clinical Medicine,

\section{Dovepress}

Journal Citation Reports/Science Edition, EMBase, Scopus and the Elsevier Bibliographic databases. The manuscript management system is completely online and includes a very quick and fair peer-review system, which is all easy to use. Visit http://www.dovepress.com/ testimonials.php to read real quotes from published authors. 